European Federation of Societies for Ultrasound in Medicine and Biology

\title{
Visit www.efsumb.org for the full provisional programme
}

\section{A few tasters below of the state-of-the-art lectures}

\section{Obstetrics CNS session \\ Thursday 11 June at 10.30}

Prenatal diagnosis of CNS anomalies is one of the main justifications for the use of ultrasound in pregnancy. The incidence of CNS anomalies in a low risk population is close to $1 \%$ and prenatal diagnosis has an important impact. The international society of ultrasound in obstetrics and gynecology (ISUOG) has published guidelines for CNS scanning, and three transverse planes are recommended for the basic scanning; the transventricular plane, the transthalamic plane and the transcerebellar plane. Aurora Røset is an expert in fetal medicine from the center for fetal medicine in Trondheim, Norway, and she will present the basic scanning through a live demonstration and also have a lecture about diagnosis and follow up of lateral ventriculomegaly.

Detection of abnormalities of the corpus callosum is challenging, as also counselling the parents when abnormalities are suspected. Professor Tullio Ghi is a leading expert from Italy, and he will give an update about ultrasound scanning of the corpus callosum.

This session will also include presentation of submitted abstracts.

The obstetrics and Gynecology sessions at EUROSON 2020 will comprise more than a full parallel session through the conference. Here is a short presentation of the sessions we have planned with top international experts.

\section{Fetal heart}

It has been shown that prenatal detection of heart defects improves the postnatal mortal- ity and morbidity, particularly for ductal dependent heart defects. Worldwide has the fetal heart been recognized as the most difficult organ to examine. To increase the prenatal detection rate, five short axis views have been suggested for the fetal heart examination. The three-vessel and three-vessel trachea views are specifically important for the detection of ductal dependent heart defects.

Professor Julene S. Carvalho is among the leading fetal cardiologists. She works at Brompton Center for Fetal Cardiology and is a consultant fetal and pediatric cardiologist at Royal Brompton \& St. George's Hospital in London, United Kingdom. She will give a live demonstration on how to examine the fetal heart and also give a presentation where she focuses on the three-vessel and three-vessel trachea views.

Associate Professor Eva Tegnander is a midwife and a sonographer with special interest in ultrasound education and the fetal heart examination. She works at the Norwegian University of Science and Technology and at the National Center for Fetal Medicine at St. Olavs Hospital in Trondheim, Norway. She will present the prenatal detection rate of congenital heart defects. This session will also include presentation of submitted abstracts.

\section{Ultrasound is the future diagnostic tool in active labour}

Traditionally, labor progress is based on clinical findings, however, clinical examinations of fetal station and position is subjective and inaccurate. Several studies have demonstrated that ultrasound is more accurate and reproducible than clinical examinations. The international society of ultrasound in obste- trics and gynecology (ISUOG) has published guidelines for intrapartum ultrasound and suggested the following indications:

- Slow progress or arrest of labor in the first stage

- Slow progress or arrest of labor in the second stage

- Ascertainment of fetal head position and station before considering or performing instrumental vaginal delivery

- Objective assessment of fetal head malpresentation

In this session, Torbjørn Eggebø will start with a practical presentation and show the transabdominal and transperinal ultrasound techniques used. Fetal lie, position, presentation, attitude and station can easily by assessed with ultrasound.

Professor Tullio Ghi from Italy is a pioneer in this field, and he will present the ISUOG guidelines and also present how to diagnose malpositions and malpresentations.

Slow progress in the second stage is very common in all delivery units, and the birth attendant should make important decisions; wait for spontaneous delivery, perform an operative vaginal delivery or do a cesarean section. Birgitte Kahrs will present clinical examples and show how ultrasound can be helpful for the clinicians in decision-making.

\section{Transvaginal ultrasound}

In this session we will focus on the use of transvaginal ultrasound in the assessment of ovarian tumors and cervical length. Professor Lill Valentin will lead us through the development of the IOTA (international ovarian tumor analysis) rules aiming to differentiate benign from malignant ovarian tumors and measurement of cervical length 
for prediction of preterm labour. Professor Nils Halvdan Morken is an epidemiologist with extensive research background on preterm birth who will present several aspects of preterm birth from a global perspective.

\section{Ultrasound for fetal growth assessment}

Ultrasound is the main tool used to assess fetal wellbeing and growth. Several growth charts are published, including two recent international collaborative longitudinal studies (Intergrowth 21 and WHO), providing reproducible and validated nomograms aiding to clinical decision making regarding fetal growth restriction and macrosomia. Two leading experts involved in these studies will discuss different views on normal fetal growth (professor Torvid Kiserud) and current guidelines on management of intrauterine growth restriction in singleton and twin pregnancies (professor Alexandros Sotiriadis).

\section{Fetal interventions}

Prenatal therapeutic interventions save lives. Blood transfusions to anemic fetuses, drainage of pleural effusion and coagulation of communicating placental vessels in monochorionic twin pregnancies with twin-twin transfusion syndromes are established procedures. Experienced therapeutic teams are necessary for achieving good results, and centralization of advanced fetal interventions is recommended, either on national or international levels. The Nordic and Baltic countries have established an international network for fetal interventions, and Karin Sundberg from the Fetal medicine department at Rigshospitalet, Copenhagen will present experiences from the first five years of this collaborating network. She will also have a lecture about drainage of fetal pleural effusion.

The ex utero intrapartum treatment (EXIT) is a surgical procedure performed in cases of expected postpartum fetal airway obstruction. Placental circulation is maintained until establishment of a patent airway. Professor Sturla Eik-Nes from the National center for fetal medicine in Trondheim introduced this technique in Norway and will present how and when to do this procedure.

\section{Placenta and umbilical cord}

The incidence of placenta accreta spectrum is increasing. The condition is associated with increased risk of adverse maternal outcome including risk of profuse bleeding and peripartum hysterectomy. Only one third to half of the cases are identified before birth, despite that ultrasound examination has a high sensitivity and specificity for identifying placenta accrete spectrum. Increased awareness of risk factors and a systematic approach to ultrasound examination of the placenta is the way to improve prenatal detection. Dr A. Bhide is a lead clinician for the Day Assessment Unit and honorary Senior Lecturer at St. George's University of London. He is also a scientific editor of BJOG and member of the editorial board for three other journals, and he will give an update to the theme, to raise our attention and improve our routine.

A normal development of the umbilical cord is crucial for a successful outcome of pregnancy, and an abnormal cord may be an indicator for increased risk. There is an increased awareness for umbilical cord accidents as it contributes substantially to intrauterine deaths. Dr C Ebbing is a senior consultant at the fetal medicine section, Department of obstetrics and gynecology and an assistant professor at the University of Bergen. Through epidemiology research based on data from the Medical Birth Registry of Norway and clinical research, she has focused on the cord and placenta, and associations to adverse outcome. She will give an overview of the theme to raise awareness and will demonstrate how to examine the cord and placenta by ultrasound.

This session will also include presentation of submitted abstracts.

\section{Doppler}

Doppler assessment of fetal and maternal circulation is a cornerstone in the screening, diagnosis and monitoring of high-risk pregnancies. G Acharya is a professor at Karolinska Hospital in Stockholm Sweden, and chief Editor in Acta Obst.Gyn Scand. He will put a light on monitoring of high-risk pregnancies and growth restriction in late pregnancy, and the use of cerebroplacental ratio or the opposite umbilico-cerebral ratio.
The fetal liver, being the organ that receives most of the umbilical return, is central in the regulation of fetal growth, and is called "the metabolic brain" of the fetus. The fetal venous circulation has traditionally been regarded as difficult to examine by Doppler. Professor J Kessler, Head of the obstetric section in Haukeland University Hospital and professor at the University of Bergen will do a demonstration of how to assess the fetal and maternal circulation, with a focus on the venous fetal liver circulation, before his lecture on the same theme.

This session will also include presentation of submitted abstracts.

\section{Emergency reception}

Ultrasound has become a key diagnostic tool in emergency medicine and ultrasound courses are introduced for physicians trained for working in emergency receptions. Lars Petter Bjørnsen from the department of anesthesiology at Trondheim University Hospital will tell us about point-of-care ultrasound in the emergency department.

Emergency situations sometimes occur during pregnancy and professor Torbjørn Eggebø from the National Center for fetal medicine in Trondheim will present the six basic steps for ultrasound in pregnancy recommended by the international society for ultrasound in obstetrics and gynecology (ISUOG). The basic steps are to examine the number of fetuses, heart activity, fetal presentation, placental location, amniotic fluid, and do basic measurements.

Ultrasound was early introduced for medical students at Haukeland University Hospital, and professor Jone Trovik will present the educational program used in Bergen. This session will also include presentation of submitted abstracts.

\section{Gastrointestinal ultrasound for guiding day-to-day clinical decisions}

Gastrointestinal ultrasound (GIUS) has received increased interest in recent years as the need of non-invasive tools for repeated investigations has escalated. EFSUMB has been 
crucial in the development the first guidelines and recommendations in this field.

In EUROSON 2020 we have two sessions dedicated to GIUS on Friday June 12. We have been lucky to recruit active and dedicated lecturers with long experience in clinical work, research and teaching.

The first session has a broad view spanning from basic scanning to multi-parametric GIUS, and from acute surgical conditions to functional disorders. GIUS is both easily accessible and handy for the surgeon and has the spatial and temporal resolution for the observation of bowel physiology.

The second session is focused on inflammatory bowel diseases presenting the recently published guidelines. In addition, special attention will be given to the subjects of detecting disease activity in ulcerative colitis and separating fibrosis from inflammation in Crohn's disease. GIUS may well be the affordable one-stop-shopping diagnostic tool in IBD.

\section{Endoscopic Ultrasound (EUS) sessions at EUROSON 2020}

Friday, June 12 in the afternoon, and Saturday, June 13 in the morning we have planned three session on endoscopic ultrasound. The topcis will be pancreatic EUS, EUS guided tissue sampling and Interventional EUS guided procedures. If EUS is your field of interest, these sessions will cover new aspects on the use of EUS elastography and contrast enhanced ultrasound in pancreatic lesions by Christoph Dietrich and Adrian Safoiu. Taking micro-biopsies from EUS guided in order to diagnose pancreatic cysts will be covered by Roald F. Havre.

In the next session on Saturday June 13 at 08.30 EUS guided tissue sampling will be in focus. We will learn about the development of todays' EUS-fine needle aspiration from the founder of EUS-FNA, Peter Vilmann. The more recent EUS biopsy needles are used more and more extensively to collect diagnostic tissue samples. What are the pros and cons of these tools? Christian Jenssen will tell us more about this. We will also have the perspective of the pathologist: What is needed for a firm tissue diagnosis? and what are the limitations of the samples provided by EUS needles?

The following session will focus on the use of EUS in guiding therapeutic procedures. We will learn about how lumen apposing stents can be used to create gastro-ileal anastomosis in the setting of gastric outlet obstruction. Dr. Pham will talk about this as well as presenting our first experiences in using EUS guided Radio-Frequency Ablations in pancreatic lesion. Dr. Vilmann will close the session by presenting the future of EUS. If you want a focused update on these central EUS topics please register for the EUROSON 2020.

Information and link to registration: http://euroson2020.org

\section{EUROSON 2020 + Liver elastography $=$ TRUE!}

At this years' EUROSON 2020 conference in Bergen several prominent names within liver and elastography research will be present. Among invited speakers Prof. Dr. Giovanna Ferraioli will hold a state-of-the-art lecture on elastography methods in liver diseases. Prof. Fabio Piscaglia will hold a lecture on US and CEUS in the diagnosis of HCC, and Prof. Chris de Korte will talk about US based steatosis quantification and the validation of these in patients. Dr. Mette Vesterhus will talk about liver elastography in patients with primary sclerosing cholangitis.
Prof. Ferraioli is a member of the editorial board of European Radiology, World Journal of Gastroenterology, Journal of Ultrasound in Medicine, International Journal of Radiology, Journal of Ultrasound, and she's a referee for more than 60 international journals. She is on several committees and is a member of EFSUMB expert team for the guidelines on liver elastography, a member of WFUMB expert panel for the update of the guidelines on liver elastography, and a member of the QIBA initiative in shear wave speed in diffuse liver diseases.

Prof. Piscaglia is professor and Consultant at the University Hospital in Bologna, Italy and former President of EFSUMB, and currently Associate Editor of EJU/Ultraschall in der Medizin and Digestive and Liver Disease.

Prof. Chris de Korte is professor in Medical Ultrasound Techniques and Medical Ultrasound imaging, Radboud University Nijmegen Medical Center, Netherlands. He has received several grants for his research. He is editorial board member of EJU/Ultrasound in Medicine and Biology, Acta Acustica united with Acustica, and the Journal of the British Medical Ultrasound Society and member of the Technical Program Committee of the International IEEE Ultrasonics Symposium.

Dr. Mette Vesterhus is Associate Professor and Consultant in Hepatology and Gastroenterology at University of Bergen and Haraldsplass Deaconess Hospital in Bergen, Norway. She is currently the President of the Norwegian Association of Gastroenterology and has a profound experience in research in chronic liver disease, particularly in Primary Sclerosing Cholangitis and the use of ultrasound in hepatology. 


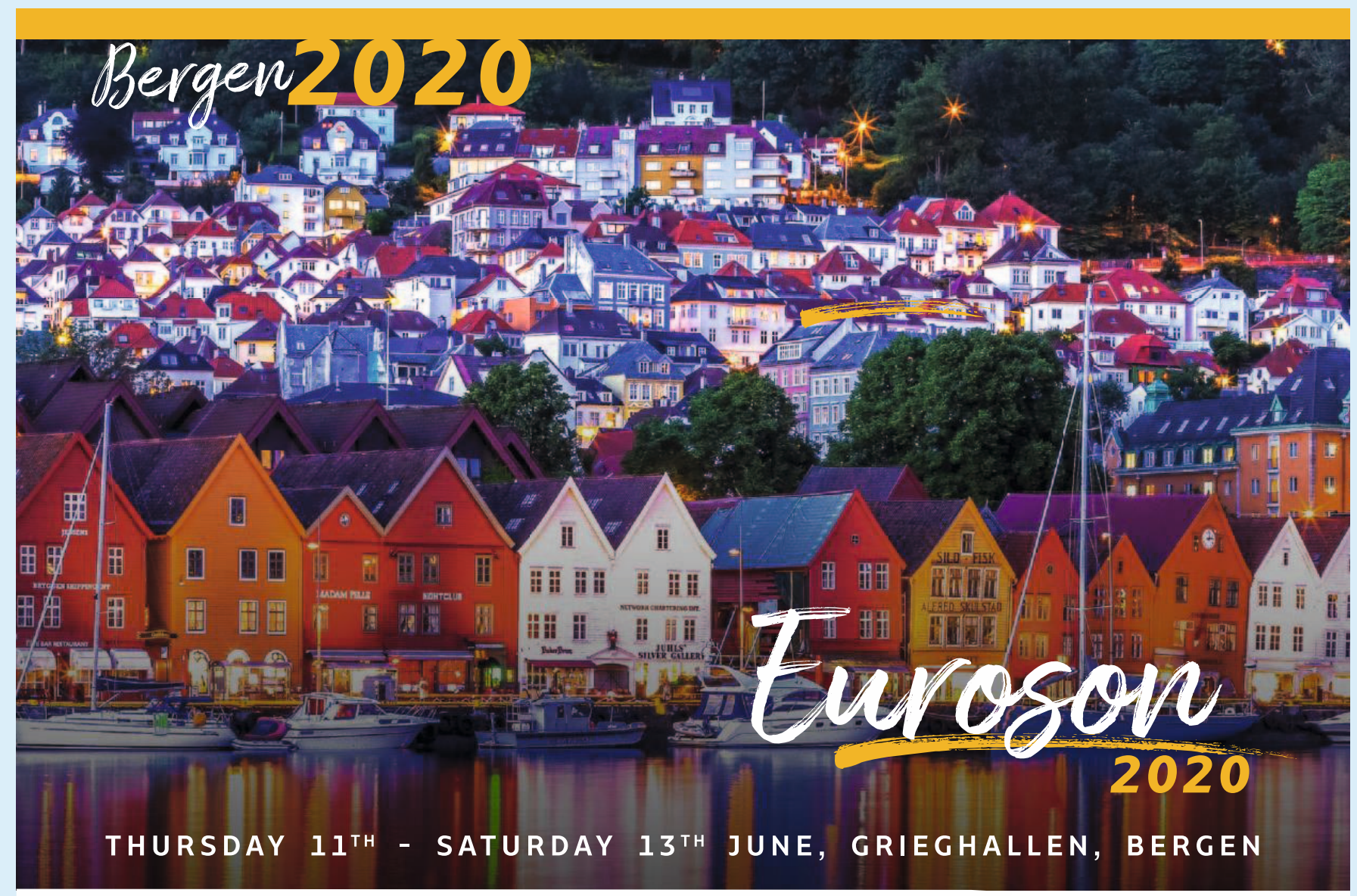

Dear colleagues,

We cordially invite you to Euroson 2020 taking place in Bergen, the gateway to the fjords and mountains of Norway. Bergen is a historic city beautifully located along the western coastline with a pulsating harbor surrounded by 7 mountains.

The venue of the congress is Grieghallen, named after Norway's famous composer Edvard Grieg, who was born and lived in Bergen. The congress is organized by the National Centre for Ultrasound in Gastroenterology and Norwegian Society for Diagnostic Ultrasound (NFUD), and this event will be NFUD's 44th conference.

Welcome to the 32nd Euroson Congress in Bergen, 11 - 13 June, 2020

Visit euroson2020.org for more information.
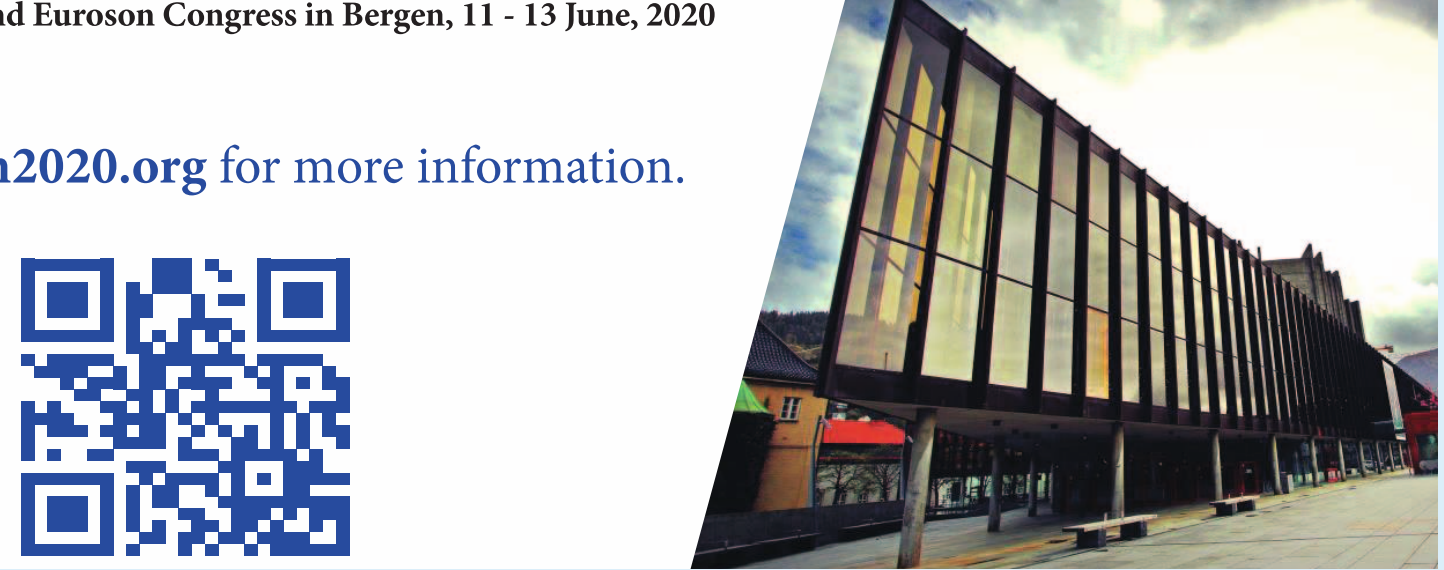